\title{
Arquitetura de Sistemas de Informação - Evolução e Análise Comparativa de Modelos
}

\author{
Tania Fatima Calvi Tait \\ Doutoranda no Programa de Pós- Graduação em Engenharia de Produção (PPGEP) \\ Universidade Federal de Santa Catarina (UFSC) - Florianópolis - SC \\ Professora do Departamento de Informática - Universidade Estadual de Maringá \\ Rua Cap. Romualdo de Barros, 965/102-A CEP 88040-600 Florianópolis - SC \\ e-mail: tania@eps.ufsc.br
}
Roberto C. S. Pacheco*
Aline França de Abreu*
* Professores do PPGEP -UFSC
e-mail:pacheco@eps.ufsc.br
e-mail: aline@eps.ufsc.br

Resumo

O termo arquitetura, tradicionalmente vinculado à área de hardware, passa a ser aplicado à área de sistemas de informaçāo, ao mesmo tempo em que são apresentados modelos ou estruturas de arquitetura de sistemas de informação (ASI) vinculados à essa transformaçăo. O presente artigo apresenta uma evoluçāo do conceito de ASI e seus enfoques e uma análise comparativa entre alguns modelos de ASI reconhecidos na literatura. Para essa análise comparativa é utilizada a combinaçāo dos elementos: visāo da organizaçāo, negócios, sistemas, tecnologia c usuários que devem compor uma ASI.

Abstract

The word archicteture has been connected to hardware, but now it is relationed to information systems area, too. This paper presents an evolution of Information Systems Architecture (ISA), their visions and a comparision between some ISA models by utilizing of elements which are: organization view, business, systems, technology and users.

Palavras-Chaves:

Arquitetura de Sistemas de Informação, Sistemas de Informaçāo, Modelos de Arquitetura de Sistemas de Informação.

Keywords:

Information Systems Architecture; Information Systems; Information Systems Architecture Models.

\section{Introdução}

A perspectiva dos sistemas de informação (SI), inicialmente utilizando o computador como ferramenta para fornecimento de dados objetivando a rapidez das tarefas rotineiras, evoluiu para uma perspectiva de negócios, que ampliou as exigências em relação aos SI, em parte, possibilitadas pelas tecnologias disponibilizadas e pelos avanços específicos em hardware e software.
Como forma de contribuir para o processo de desenvolvimento e implantação de SI, neste cenário, surge a abordagem da Arquitetura de Sistemas de Informação (ASI).

Inicialmente tratada como arquitetura de dados, a ASI acompanha a evolução dos SI e encampa uma visão mais abrangente, com a inclusão da perspectiva de negócios, a visão organizacional, os próprios SI, a tecnologia disponível e os usuários envolvidos (Tait et al, 1998). 


\section{PRODUÇÃO}

As pesquisas em ASI têm evoluído com a apresentação de modelos de ASI com enfoques diferenciados (Zachman, 1987; Scheer, 1992; Stecher, 1993; Inmon et al, 1997, entre outros). No Brasil, contudo, a pesquisa sobre ASI tem-se apresentado incipiente e os trabalhos, de modo geral, envolvem: a integração entre organização, negócios, sistemas, tecnologia e usuários (Tait, 1994); a apresentação de ferramenta de ASI (Godoy, 1996); e a arquitetura tecnológica (Raskin, 1997).

Dentro deste contexto, visando uma compreensão mais adequada da evolução da ASI, são apresentados, neste artigo, o seu conceito e evolução, seus diversos enfoques e uma análise comparativa de alguns modelos de ASI, consolidados na literatura.

\section{Conceito e evolução da ASI}

No final dos anos 80, o termo arquitetura, vinculado à área de hardware, passa a ser utilizado na área de software (Zachman, 1987) e (Richardson et al, 1990), considerando toda a estrutura dos sistemas de informação (SI), desde o planejamento estratégico até $\mathrm{o}$ armazenamento de dados, inclusive com a vinculação da ASI com a estratégia de SI (Loftin, 1989).

Associada à evolução do termo arquitetura, que pode ser observada no quadro 01 , uma série de interpretações começa a surgir e, a arquitetura passa a ser considerada em quatro visões básicas: arquitetura de dados; arquitetura tecnológica (Earl, 1989; Laudon \& Laudon, 1996); arquitetura voltada para os negócios (Cook, 1996); e arquitetura abrangente (Zachman, 1987; Scheer, 1992; Ryan \& Santucci, 1993; Kim \& Everest, 1994), refletindo conceitos abordados em várias pesquisas.

Contudo, o conceito que mais tem se destacado é o de arquitetura abrangente, que coloca a ASI como o estabelecimento de um conjunto de elementos cuja finalidade é proporcionar um mapeamento da organização no tocante aos elementos envolvidos com o processo de desenvolvimento/implantação de SI.

Nesta linha de conceito abrangente, Richardson et al (1990) afirmam que uma arquitetura não deve ser vista como uma solução formal para todo problema tecnológico, visto que a meta da. arquitetura é interrelacionar dados, deixar disponível hardware, software e recursos de comunicação e ter o staff para efetivamente processar transações, produzir informação e suportar uma variedade de domínios de atividade humana.

Como benefícios da ASI, Cook (1996) coloca, também, que a mesma: dinamiza processos de negócios; reduz complexidade dos sistemas; capacita integração na empresa através do compartilhamento de dados e capacita a evolução mais rápida para novas tecnologias.

Zachman (1998) salienta, ainda, que toda empresa que pretende ser uma jogadora na era da informação deve ter em mente a elaboração de uma ASI e que a revolução arquitetural é iminente, pois, hoje, a informação é valorizada nas organizações e existem ferramentas automatizadas que facilitam a elaboração de uma arquitetura.

Quadro 01. Evolução do termo arquitetura.

\begin{tabular}{|l|ll|}
\hline \multicolumn{1}{|c|}{ Período } & & \multicolumn{1}{c|}{ Significado } \\
\hline Até anos 80 & $\bullet$ & arquitetura tradicional associada com projeto de hardware \\
\hline 1987 & $\bullet$ & arquitetura passa a ser associada à área de software \\
\hline Anos 90 & $\bullet$ & arquitetura expressa SI \\
& $\bullet$ & arquitetura associada às estratégias de negócios \\
\hline Final dos anos 90 & $\bullet$ & arquitetura de SI como arquitetura de informação da empresa \\
\hline
\end{tabular}


Enfim, a ASI possibilita como contribuições básicas: aprimorar as atividades do planejamento estratégico de SI; melhorar o desenvolvimento de SI computadorizados; racionalizar a execução das atividades; economizar tempo; estabelecer ordem e controle no investimento de recursos de SI; definir e interrelacionar dados; fornecer clareza para a comunicação entre os membros da organização; permitir melhorar e integrar ferramentas e metodologias de desenvolvimento de software; estabelecer credibilidade e confiança no investimento de recursos do sistema; fornecer condições para aumentar a vantagem competitiva.

\section{Enfoques dados à ASI}

As diferentes visões de ASI, descritas no item anterior, dão suporte tanto para discussões como para a elaboração de modelos de ASI, configurando-se em uma diversificação de enfoques, os quais podem ser observados no quadro 02 . organização (Zachman, 1987, Scheer, 1992 e 1997 ; Sowa \& Zachman, 1992; Kim \& Everest, 1994), extrapolando a visão de dados ou tecnologia. Aqui, também se enquadram a utilização da abordagem da estrutura de ASI de Zachman para a implementação de datawarehouse e datastore (Inmon et al, 1997) e a arquitetura específica, encontrada em Stecher (1993), que apresenta a Retail Application Architecture (RAA), voltada para integrar dados e aplicações em uma empresa varejista. Uma abordagem no tratamento de ASI como suporte a toda atividade de desenvolvimento de sistemas, devendo ser produzida antes de iniciar qualquer atividade, é dada por Inmon e Caplan (1992). Nesta arquitetura, o projeto pode incluir uma arquitetura de dados, uma arquitetura de aplicação e uma arquitetura técnica.

Para Earl (1989), a estrutura tecnológica, a qual chama arquitetura, compreende quatro elementos: a computação (hardware e software), as comunicações (redes de telecomunicações), os dados (relacionamentos entre eles) e aplicações.

Quadro 02. Visões de ASI.

\begin{tabular}{|l|l|}
\hline \multicolumn{1}{|c|}{ Pesquisadores } & \multicolumn{1}{|c|}{ Preocupação } \\
\hline $\begin{array}{l}\text { Zachman (1987); Sowa \& Zachman (1992); } \\
\text { Kim \& Everest (1994); Ryan \& Santucci (1993); } \\
\text { Scheer (1992) e Scheer (1997) }\end{array}$ & ASI abrangente \\
\hline Earl(1989); Laudon \& Laudon (1996) & Infra-estrutura tecnológica \\
\hline Kosanke \& Klevers (1990) & Manufatura (CIM-OSA) \\
\hline IFIP.WG; Inmon \& Caplan (1992) & Ciclo de vida do SI \\
\hline Stecher (1993) & Aplicação especifica \\
\hline Cook (1996) & Visão de negócios \\
\hline Inmon et al(1997) & ASI abrangente, suporte para datawarehouse \\
\hline
\end{tabular}

Para uma compreensão adequada da situação das pesquisas em ASI torna-se conveniente $o$ conhecimento desses enfoques, agrupados em: arquitetura abrangente; desenvolvimento de sistemas; arquitetura e TI e, arquitetura e negócios.

$\mathrm{Na}$ linha de arquitetura abrangente estão as pesquisas que trabalham com a integração dos SI e
Na visão de Laudon e Laudon (1996), a arquitetura de informação é a forma particular que a TI tem, em uma organização, de alcançar metas selecionadas ou funções, estando estritamente ligada ao aspecto tecnológico, pelo arranjo de hardware, software, dados e redes de computadores, na linha seguida por Earl (1989). 


\section{PRODUÇÃO}

Cook (1996) considera que a visão dos

negócios de ASI fornece um conjunto conceitual de SI e as ligações entre estes SI, e que a abordagem para a construção de uma arquitetura de informação focaliza em dados ou processos.

O estudo dos diferentes enfoques mostrou que: 1. a estrutura de Zachman (1987), principalmente por ser a pioneira na discussão de arquitetura de sistemas como fator abrangente, extrapolando a dimensão de dados ou tecnologia, tem servido de base para diversos pesquisadores (Sowa \& Zachman, 1992; Stecher, 1993; Kim e Everest, 1994; Cook, 1996 e Inmon et al, 1997); 2. a visão de arquitetura como tecnológica continuà sendo elaborada (Laudon \& Laudon, 1996; Raskin, 1997); e; 3. alguns modelos de ASI para aplicações específicas (Stecher, 1993), consideram a visão abrangente em sua estruturação.

\section{Modelos de ASI}

Os modelos de ASI ou estruturas apresentados a seguir foram selecionados com base na importância que têm para a construção de ASI desde os primórdios do surgimento de pesquisas na área e na visão de ASI abrangente que encampa, minimamente, aspectos organizacionais, de negócios e de sistemas.

\subsection{Estrutura de Zachman}

A estrutura de ASI proposta por Zachman (Zachman, 1987, Sowa \& Zachman, 1994;

Zachman, 1996; Inmon et al, 1997) é uma abordagem amplamente utilizada para desenvolver e/ou documentar uma ASI para a empresa, com a finalidade de fornecer uma estrutura básica que suporta: a organização, o acesso, a integração, a interpretação, o desenvolvimento, o gerenciamento e a troca de um conjunto de representações de ASI nas organiza- ções. São apresentados, na estrutura de Zachman, os elementos: escopo, modelo de negócios da empresa; modelo de sistema; modelo de tecnologia e componentes, os quais são cruzados com os elementos dados, função, rede, acrescidos de pessoas, tempo e motivação por Sowa \& Zachman (1992).

\subsection{Modelo de Gifford}

Gifford (1992) propõe uma ASI que aplique a plataforma de hardware adequada à atividade dos negócios, formada por três dimensões: os componentes; o ciclo de desenvolvimento e a correlação entre as várias plataformas. Dentro dos componentes devem ser modelados: os dados (Diagrama Entidade-Relacionamento - DER) e funções, associados ao hardware necessário. A correlação entre as várias plataformas envolve o hardware, o sistema operacional, os protocolos de comunicação de dados e, as ferramentas de aplicações e de bancos de dados.

\subsection{Arquitetura Aris}

A arquitetura ARIS (Architecture of Integrated Information Systems) (Scheer, 1992), constitui uma estrutura na qual os SI integrados possam ser desenvolvidos, otimizados e convertidos em implementações técnicas EDP (Electronic Data Processing). Funções, organização, dados e controle compõem a arquitetura ARIS.

Para a arquitetura ARIS, que usa como modelo para a modelagem dos dados, a abordagem ERM (entidade-relacionamento) estendido, é necessário escolher uma linguagem descritiva e uniforme que seja aplicada a todos os blocos. O modelo da cadeia de processo é tomado como ponto inicial para o desenvolvimento da arquitetura. As funções, na ARIS, descrevem o processo de transformação da informação e são consideradas do ponto de vista 
de sua estrutura de função, sua seqüência de processamento e seu suporte, usando modelos de decisão.

\subsection{Arquitetura IFIP.WG}

A estrutura, descrita na forma de modelo de informação, apresentada por Olle e outros (Scheer. 1992), consiste de dois componentes fundamentais: as perspectivas e os níveis de um ciclo de vida de um SI. As visões são diferenciadas em: orientada a dados, orientada a processos e orientada a procedimentos. A criação destas visões é baseada em métodos conhecidos de desenvolvimento de SI.

Nesta metodologia, três estágios no processo de desenvolvimento de SI foram selecionados: planejamento de SI, análise de negócios e projeto de sistemas. O planejamento de SI se refere ao planejamento estratégico de SI. No contexto da análise de negócios, um sistema de informação existente para uma empresa toda ou para alguma sub-área é analisado. O SI é criado no estágio de projeto de sistemas.

\subsection{Arquitetura CIM-OSA}

A arquitetura CIM-OSA mistura questões metodológicas gerais de SI e aspectos específicos de aplicações CIM (Computer Integrated Manufacturing). Trabalha com as visões de organização, recurso, informação e função.

Segundo Kosanke e Klevers (1990), a meta do CIM-OSA é fornecer uma arquitetura de sistema aberto (OSA - Open System Architecture) que: cubra todas as necessidades de informação interna e externa em uma empresa de manufatura; suporte todos os níveis de gerenciamento em seu planejamento estratégico, tático e operacional, bem como a operação direta de prọ̣eto do produto, manufatura etc; e, suporte os processos de tomada de decisão pelo fornecimento de informação necessária.

\section{Análise Comparativa dos Modelos de ASI} Apresentados

Os modelos de ASI são analisados a partir da visão de arquitetura abrangente, configurada na visão mais atual de ASI e que inclui os elementos organização, negócios, sistemas, tecnologia e usuários (Tait et al, 1998). O quadro comparativo (03) sintetiza esta análise.

O estudo dos modelos e estrutura de ASI proporcionou o levantamento de algumas lacunas que justificam o aperfeiçoamento das estruturas ou criação de novos modelos: a visão de usuários; a cultura organizacional; os tipos de SI; os tipos de empresas e a integração com desenvolvimento de software.

O aspecto usuário é considerado pelos modelos apresentados de forma simplista, ora como participante nas etapas iniciais do processo ora como classificação para a segurança dos sistemas.

As estruturas pautadas por uma visão mais abrangente vão além desta visão simplista e incluem aspectos de motivação para os participantes usuários. Não são realizadas, também, abordagens adequadas na questão do usuário, ignorando-se aspectos de interface humano-computador; as especificidades da organização e formas de participação dos usuários diante das novas tecnologias disponibilizadas.

Mais abrangente que o aspecto usuário tem-se a cultura organizacional, importante, à medida em que mostra a existência de diversas "culturas" operando na empresa: a cultura gerencial, a cultura da base operacional, a cultura de grupos, a cultura de trabalhadores e a cultura da empresa modificada pela cultura local (Moscovici, 1993), cujos aspectos podem interferir no desenvolvimento das atividades na organização, inclusive no desenvolvimento e 
Quadro 03. Quadro comparativo de modelos de ASI.

\begin{tabular}{|c|c|c|c|c|c|}
\hline $\begin{array}{l}\text { Modelos/ } \\
\text { Elementos }\end{array}$ & $\begin{array}{l}\text { Estrutura de } \\
\text { Zachman }\end{array}$ & Modelo Gifford & Arquitetura Aris & $\begin{array}{l}\text { Modelo } \\
\text { IFIP.WG }\end{array}$ & CIM-OSA \\
\hline Objetivos & \begin{tabular}{|l|} 
Fornece uma forma \\
de ver um sistema de \\
diferentes \\
perspectivas e \\
considera como \\
finalidade de uma \\
ASI, a demonstração \\
de como tudo \\
funciona em \\
conjunto
\end{tabular} & $\begin{array}{l}\text { Oma ASI que } \\
\text { aplique à } \\
\text { plataforma de } \\
\text { hardware }\end{array}$ & $\begin{array}{l}\text { Estrutura para } \\
\text { integrar SI }\end{array}$ & $\begin{array}{l}\text { Perspectivas e } \\
\text { níveis de ciclo de } \\
\text { vida de um SI. }\end{array}$ & $\begin{array}{l}\text { Questões } \\
\text { metodológicas } \\
\text { gerais de SI e } \\
\text { aplicações CIM }\end{array}$ \\
\hline Visões & $\begin{array}{l}\text { Escopo, modelo de } \\
\text { negócios da } \\
\text { empresa; modelo de } \\
\text { sistema; modelo de } \\
\text { tecnologia e } \\
\text { componentes. }\end{array}$ & $\begin{array}{l}\text { Dados e funções } \\
\text { associados ao } \\
\text { hardware } \\
\text { necessário }\end{array}$ & $\begin{array}{l}\text { Organização, } \\
\text { dados, } \\
\text { controle e } \\
\text { função }\end{array}$ & $\begin{array}{l}\text { Dados, } \\
\text { processos e } \\
\text { procedimentos }\end{array}$ & $\begin{array}{l}\text { Organização, } \\
\text { recurso, } \\
\text { in formação e } \\
\text { função }\end{array}$ \\
\hline Organização & $\overline{-}$ & - & $\begin{array}{l}\text { Definição das } \\
\text { necessidades; } \\
\text { especificação do } \\
\text { projeto; } \\
\text { descrição da } \\
\text { implementação }\end{array}$ & Não apresenta & $\begin{array}{l}\text { Permite estruturar } \\
\text { as diferentes } \\
\text { responsabilidades } \\
\text { na empresa }\end{array}$ \\
\hline Negócios & $\begin{array}{l}\text { Projeto de negócios, } \\
\text { entidades de } \\
\text { negócios e processos } \\
\text { e sua interação }\end{array}$ & $\begin{array}{l}\text { Plataforma } \\
\text { adequada às } \\
\text { atividades de } \\
\text { negócios }\end{array}$ & $\begin{array}{l}\text { Mostra diferentes } \\
\text { graus de } \\
\text { proximidade de } \\
\text { TI }\end{array}$ & $\begin{array}{l}\text { Definição das } \\
\text { necessidades dos } \\
\text { SI }\end{array}$ & $\begin{array}{l}\text { Início do } \\
\text { processo; } \\
\text { definição das } \\
\text { necessidades dos } \\
\text { negócios }\end{array}$ \\
\hline Sistemas & \begin{tabular}{|l|} 
Modelo projetado \\
pelos analistas de \\
sistemas; \\
elementos de dados \\
e funções que \\
representam \\
entidades de \\
negócios e processos
\end{tabular} & Ciclo de vida & $\begin{array}{l}\text { Aplicação; } \\
\text { sistemas } \\
\text { integrados; } \\
\text { usa E_R }\end{array}$ & $\begin{array}{l}\text { Planejamento de } \\
\text { SI; } \\
\text { análise de } \\
\text { negócios e } \\
\text { projeto; } \\
\text { usa modelo E-R }\end{array}$ & $\begin{array}{l}\text { Comunicação } \\
\text { intrasistemas }\end{array}$ \\
\hline Tecnologia & $\begin{array}{l}\text { Detalhes das } \\
\text { linguagens de } \\
\text { programação; } \\
\text { dispositivos de } \\
\text { entrada e saída }\end{array}$ & $\begin{array}{l}\text { Hardware; } \\
\text { sistema } \\
\text { operacional; } \\
\text { protocolos de } \\
\text { comunicação e } \\
\text { dados e } \\
\text { ferramentas de } \\
\text { aplicação e banco } \\
\text { de dados }\end{array}$ & Estrutura física & - & $\begin{array}{l}\text { Suporte para } \\
\text { integração física } \\
\text { dos sistemas }\end{array}$ \\
\hline Usuários & Quem faz o trabalho & - & $\begin{array}{l}\text { Função; } \\
\text { classificação }\end{array}$ & - & $\begin{array}{l}\text { Necessidades } \\
\text { definidas pelos } \\
\text { usuários }\end{array}$ \\
\hline $\begin{array}{l}\text { Desenvolvi- } \\
\text { mento de } \\
\text { software }\end{array}$ & $\begin{array}{l}\text { Modelagem de } \\
\text { dados }\end{array}$ & $\begin{array}{l}\text { Planejamento, } \\
\text { análise, projeto, } \\
\text { implementação e } \\
\text { suporte }\end{array}$ & $=$ & - & - \\
\hline
\end{tabular}


implantação de SI.

Para se ter uma idéia da relevância da consideração dos tipos de SI e a importância para o seu desenvolvimento e implantação, estudos (Zachman, 1996) consideram que a maioria das empresas ainda possui uma grande quantidade de sistemas não integrados, construídos de qualquer forma e que consomem recursos financeiros, humanos e de tempo em manutenções intermináveis. Muitos ainda enquadrados como sistemas transacionais ou legados.

As estruturas de ASI apresentam-se como ideais para qualquer tipo de empresa. À exceção de poucas estruturas, direcionadas a empresas específicas, a exemplo de Stecher (1993), a maioria desconsidera aspectos particulares encontrados nas diversas estruturas organizacionais.

A integração com o desenvolvimento de software é um aspecto importante, na medida em que são os software que suportam as atividades dos SI e, em essência, ditam a forma como os processos serão desenvolvidos a partir da implantação dos software. Ignorar o desenvolvimento dos software na questão dos SI implica em dissociar os procedimentos de sua execução.

\section{Considerações finais}

Em sua evolução, a ASI procura acompanhar as novas exigências dadas aos SI, marcadas pela transformação do conceito de informação para conhecimento e pelo uso de tecnologias para garantir a confiabilidade das informações.

A passagem do enfoque de arquitetura tecnológica para arquitetura de sistemas de informação implica em uma visão do conjunto da organização e das informações que transitam pela mesma, desde o nível operacional até o nível executivo.

Neste cenário, a estruturação de uma ASI, em seu conceito mais abrangente, incluindo as visões de organização, negócios, sistemas, tecnologia e usuários, se torna preponderante pois fornece um mapeamento da organização no tocante aos elementos envolvidos no processo de desenvolvimento/ implantação de um sistema de informação.

Esta estruturação, contudo, deve considerar as questões específicas de cada organização, as quais são imprescindíveis para o conhecimento da organização e para a identificação de barreiras e oportunidades na implantação de um modelo de ASI.

\section{Bibliografia}

COOK, M. Building Enterprise Information Architectures - Reengineering Information Systems. Ed. Prentice Hall, USA: 1996.

EARL, M. J. Strategies for information Technology. Grã-Bretanha: Prentice Hall, 1989. cap. 1 ao 5 .

GIFFORD, R. Implementing the IS Architecture. Information Systems Management, vol. 9, nr.4, pp.41-47, Fall/1992.

GODOY, F. A. R. de. O desenvolvimento de uma arquitetura de sistemas de informação para integrar os planejamentos estratégicos de negócios, tecnologia e sistemas de informação. Mestrado em Ciência da Computação, Universidade Federal de São Carlos, 1996.

INMON, W. H. \& CAPLAN, J. H. Information Systems Architecture- Development in the 90's. QED Publishing Group, Wellesley, 1992.

INMON, W.H.; ZACHMAN, J. A. 7 GEIGER, J.G. Data Stores, Data warehousing and the Zachman Framework - Manging enterprise 
PRODUÇÃO

knowledge. McGraw-Hill, USA: 1997.

KIM, Y.\& EVEREST, G. C. Building an IS architecture - coletive wisdom from the field. Information \& Management, nr. 26, 1994, pp 1-11.

KOSANKE, K. \& KLEVERS, T. CIM_OSA: architecture of enterprise integration - a report on current developments. Vol.3, nr.01, Butterworth\&Cpo Ltd, fevereiro/1990.

LAUDON, K.\& LAUDON, J. Management Information Systems-Organization and Technology. Macmillan Publishing Company, EUA, 1996, 818 págs.

LOFTIN, R. The role of information system architecture in competitive strategy. In Development the World Class Information Systems Organizations. The Dooley Group. QED Information Sciences. Inc, 1989, pp. 187-203.

MOSCOVICI, F. Renascença organizacional a revalorização do homem frente à tecnologia para o sucesso da nova empresa. 3.ed, José Olímpio Editora, Rio de Janeiro: 1993, 129 págs.

RASKIN, S. Uma arquitetura de tecnologia de informação. Anais do XXV Seminário Nacional de Informática Pública, Salvador, Bahia, 1997.

RICHARDSON, G.; JACKSON, B.M.\& DICKSON, G. W. A. Principles-Based Enterprise Architecture: Lessons from Texaco and Star Enterprise. MIS/Quartely, vol.14, nr.4, dezembro/ 1990, pp. 385-403.

RYAN, H. \& SANTUCCI, J. Building an enterprise information architecture. Enterprise Computing, 22/03/93, pp. 57-60.
SCHEER, A. Architecture of Integrated Information Systems Foundations of Enterprise Modelling, Germany, 1992.

SCHEER, A. ARIS Mentor. CD-ROM, 1997.

SOWA, J.F.\& ZACHMAN, J.A. Extending and formalising the framework for information systems architecture. IBM Systems journal, vol.31, nr.3, 1992, pp. 590-616.

STECHER, P. Building Business and application systems with the Retail Application Architecture. IBM System Journal, vol.32, nr.2, 1993, pp. 278-306.

TAIT, T.F.C. Uma avaliação do processo de planejamento estratégico de sistemas de informação em empresas do mercado brasileiro e uma proposta simplificada de arquitetura de sistemas de informação. Dissertação de Mestrado em Ciência da Computação, Universidade Federal de São Carlos, SP. 1994.

TAIT, T.F.C.; BARCIA, R.M.\& PACHECO, R. Uma arquitetura de sistemas de informação para integrar aspectos técnicos e organizacionais nos sistemas de informação. Anais do XVIII Encontro Nacional de Engenharia de Produção e IV Congresso Internacional de Engenharia Industrial, Niterói: 1998.

ZACHMAN, J.A. A framework for Information Systems Architecture. IBM System Journal, vol. 26, nr.3, 1987, pp. 276-285.

ZACHMAN, J. A. The framework for enterprise architecture: bacground, description and utility. Zachman International, 1996. Enviado por e- 
mailem22/agosto/1998 por zifa@zifa.com.br.

ZACHMAN, J. A. Enterprise architecture:

looking back and looking ahead. Database

Newsletter, maio de 1998. Enviado por e-mail em

22/agosto/1998. 